\title{
COMPARACIÓN DE ALGORITMOS DE APRENDIZAJE AUTOMÁTICO PARA LA CLASIFICACIÓN DE POSTURAS DE LA MANO Y DEDOS
}

\author{
Enaitz Otazua, Eloy Irigoyen \\ Grupo de Investigación de Control Inteligente, Universidad del País Vasco (UPV/EHU), Alameda Urquijo, \\ 48013, Bilbao, eotazua95@gmail.com \\ Eukene Imatz-Ojanguren, Thierry Keller \\ TECNALIA, Astondo Bidea, Edificio 700, 48160, Derio, eukene.imatz@tecnalia.com
}

\begin{abstract}
Resumen
El deterioro de la movilidad de la extremidad superior es una de las consecuencias más comunes de los accidentes cerebrovasculares. La estimulación eléctrica funcional (FES) ha demostrado ser una terapia efectiva para recuperar la movilidad y la función de la mano. Para desarrollar un sistema de control para la aplicación de FES es necesario primero obtener un clasificador capaz de identificar los movimientos de la mano y los dedos. Este estudio contribuye a la fase inicial de este proceso, donde se analiza el comportamiento de diferentes algoritmos durante la clasificación de gestos de la mano durante movimientos de agarre realizados por un voluntario sano. Entre los algoritmos de clasificación utilizados para el reconocimiento del movimiento de agarre, los que mejores resultados han obtenido observando la precisión y tiempo de computación han sido el Extra Trees, Random Forest y SVM.
\end{abstract}

Palabras clave: Clasificación, aprendizaje automático, mano, dedos, sensores inerciales, sensores vestibles.

\section{INTRODUCCIÓN}

El accidente cerebrovascular (ACV) normalmente resulta la parálisis de una parte del cuerpo hemiplejia - [12]. Las consecuencias más comunes del ACV son la falta de coordinación, la falta de control motor y la pérdida de movimiento funcional, limitando así la realización de actividades de la vida diaria [16]. Se ha observado que los músculos del miembro superior involucrados en movimientos de agarre sufren un déficit en el control muscular; lo cual acarrea a un deterioro de dichos músculos [12], [18], [19], [28]. Los movimientos del miembro superior también pueden verse afectados debido a los patrones aprendidos por los músculos espásticos [15], [21], [30]. Entre las terapias de rehabilitación para recuperar la movilidad del miembro superior está la estimulación eléctrica funcional (FES por sus siglas en inglés), una de las terapias más efectivas para mejorar la capacidad de movimiento voluntario en las personas que padecen una discapacidad de la extremidad superior causada por un ACV [26], [32]. FES consiste en la aplicación de estímulos eléctricos por medio de electrodos colocados sobre la piel para excitar fibras nerviosas que provocan la contracción muscular y por lo tanto un movimiento funcional como el agarre en el miembro superior.

Ante la complejidad del sistema biológico es necesario realizar un buen control del sistema FES para conseguir funciones del miembro superior como el agarre, siendo para ello de gran importancia conocer la posición de la mano en cada instante. Además, la variabilidad entre las personas, los numerosos parámetros FES y la importancia del área de estimulación para la obtención de movimientos concretos, hacen que también sea importante conocer la posición de la mano conseguida al modificar cada uno de los parámetros FES en el proceso de calibración. Por ello, y para permitir el control automático de un sistema FES con pacientes neurológicos, es necesario clasificar los movimientos de la mano inducidos por el FES en dichos pacientes. Para ello es indispensable conseguir primero un clasificador capaz de identificar los movimientos de la mano y los dedos de personas sanas.

Un clasificador de movimientos de la mano se basa en el reconocimiento de dicha actividad. Actualmente existen tres tecnologías principales para esta aplicación: basadas en la visión, en el entorno y en sensores vestibles [8], [22], [23]. En la categoría de las tecnologías basadas en visión se encuentran las cámaras, y un ejemplo de las tecnologías basadas en el entorno es el de las casas inteligentes. Debido a la miniaturización y asequibilidad, los sensores vestibles, especialmente los sensores inerciales (IMUs por sus siglas en inglés) y los acelerómetros, se han convertido de gran interés. El uso de sensores vestibles permite recoger los datos de los 
movimientos sin necesidad de forzar al usuario a estar frente una cámara $-\mathrm{y}$ los problemas de privacidad consiguientes que es to puede acarrear- o a interactuar con objetos específicos [22], [23]. A raíz de ello, muchos trabajos de reconocimiento de actividad humana se basan en el uso de sensores vestibles [1], [6], [8], [11], [23], [31], [36].

La colocación de estos sensores es un tema de gran relevancia. Para el reconocimiento de actividades como andar, sentar, correr, etc. se suelen colocar en diferentes partes del cuerpo: cintura, tobillo, torso, muslo y tronco. Sin embargo, esta colocación no da mucha información sobre el movimiento realizado con la mano, y en concreto con los dedos; colocar los sensores en la muñeca y en los dedos puede ayudar en el reconocimiento de los gestos de la mano [5].

Varios algoritmos de clasificación como el árbol de decisiones, $\mathrm{K}$ vecinos más cercanos, support vector machine, perceptrón multicapa, random forest, extra trees, agregación de bootstrap y potenciación de gradiente han sido utilizados en diversos estudios de clasificación de movimientos de la mano [4], [8], [11], [22], [25], [27]; en estos estudios se analizan los movimientos de la mano en actividades del día a día: andar, correr, comer, etc.

En el presente trabajo, se han utilizado dichos algoritmos para clasificar los distintos gestos y movimientos primitivos presentes en la función de agarre, y se ha realizado una comparación de la capacidad de reconocimiento de gestos de la mano que tienen dichos algoritmos. Para ello, se han analizado la precisión y el tiempo de computación de los diferentes algoritmos de aprendizaje automático para la clasificación de movimientos voluntarios de la mano realizados por una persona sana.

\section{MATERIAL Y MÉTODOS}

\subsection{MATERIAL}

En este estudio se han analizado diferentes movimientos realizados con la mano los cuales contribuyen en el movimiento de agarre. Para ello se han utilizado sensores inerciales; dispositivos electrónicos que miden la aceleración, la gravedad y la velocidad angular mediante los acelerómetros y giroscopios que lo integran [35]. Con el objetivo de recoger los movimientos de la mano que contribuyen en el movimiento de agarre, se han utilizado 5 IMUs (Bosch BNO055) de 9 grados de libertad, los cuales se han colocado en la muñeca, en el dedo pulgar, en el dedo índice, en el dedo medio y en el dedo anular por medio de anillos textiles elásticos tal como se observa en la figura 1.
De los IMUs colocados en los dedos índice, medio, anular y pulgar, se ha extraído la aceleración en dos de los ejes, ya que el tercer eje (horizontal respecto a las yemas de los dedos) no es relevante durante el agarre. Del IMU colocado en la muñeca se han extraído tanto la aceleración como la velocidad angular de los tres ejes.

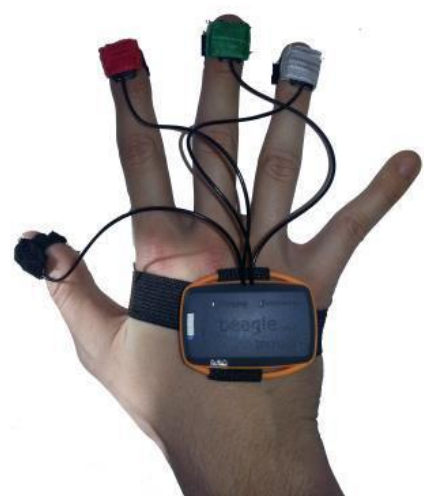

Figura 1: Sistema de sensores inerciales.

\subsection{RECOGIDA DE DATOS}

La recogida de datos se ha realizado con la mano en posición de pronación, para lo cual se ha utilizado un sistema de bloqueo de posición de antebrazo como se observa en la figura 2.

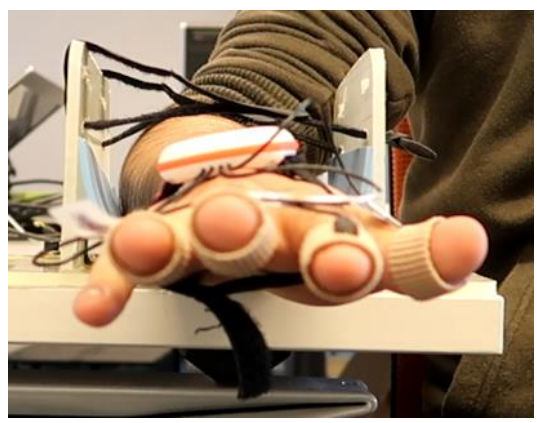

Figura 2: Sistema de bloqueo de posición del antebrazo.

Los movimientos realizados durante la recogida de datos han sido los siguientes:

- extensión y flexión de la muñeca, dedo índice y dedos medio-anular.

- aducción, abducción, extensión, oposición con el dedo meñique y oposición con flexión, del dedo pulgar en el orden escritos.

- Agarre de pinza con los dedos pulgar e índice, agarre de pinza con todos los dedos de la mano, y agarre de un bolígrafo. 
Se han realizado tres repeticiones de cada grupo de movimientos y se ha repetido este proceso en dos ocasiones. De este modo, se han grabado 22 minutos de distintas posiciones de mano obtenidas de movimientos simples y combinados (Figura 3). A1 segmentar los datos recogidos en ventanas de tiempo de 0.25 segundos con una superposición de $50 \%$ se han obtenido 6387 muestras para el entrenamiento, 2738 para el testeo de movimientos primitivos y 1067 para el testeo de movimientos complejos movimientos de agarre-.

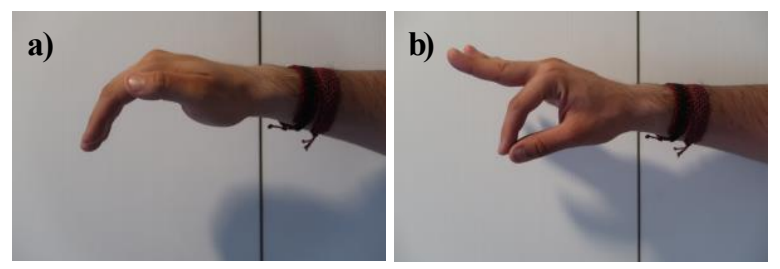

Figura 3: Ejemplos de movimiento simple (a) y movimiento combinado (b).

El etiquetado de los movimientos se ha realizado manualmente haciendo uso de las marcas temporales añadidas en la recogida de datos y los videos grabados de los movimientos de la mano.

\subsection{CLASIFICACIÓN}

Teniendo en cuenta que el agarre es causado por una combinación de movimientos de dedos y muñeca, y que el dedo medio, anular y meñique se mueven de manera coordinada en la mayoría de los casos, en el presente trabajo se han diseñado 4 clasificadores: muñeca, dedo índice, dedos medio-anular y pulgar.

Ilustrándose en el libro de fisioterapia Fisiologia articular - Miembros superiores [17] las posiciones que se han clasificado en cada clasificador son las siguientes:

- Muñeca: Extensión, posición neutra y flexión. -3 posiciones-.

- Dedo pulgar: posición neutra, aducción, abducción, extensión, oposición con el dedo meñique y oposición con flexión.

-6 posiciones-.

- Dedo índice: Extensión (medio/completo), posición neutra, y flexión.

-3 posiciones-.

- Dedos medio-anular: Extensión (medio/ completo), posición neutra, y flexión.

-3 posiciones -.

Estos clasificadores de movimientos primitivos se han utilizado posteriormente para clasificar un movimiento complejo como es el del agarre.

\subsection{ALGORITMOS DE CLASIFICACIÓN}

Una vez recogidos los datos de los movimientos realizados y segmentarlos en ventanas de 0.25 segundos [13] con una superposición del 50\% [33], lo siguiente ha sido, por un lado, etiquetar cada ventana el relación a cada clasificador, y por otro, extraer las características más relevantes de los datos recogidos y normalizarlas. Los algoritmos inteligentes de clasificación hacen posteriormente uso de dicha información para realizar la clasificación.

Las características que se han extraído, ilustrándose en otros estudios de reconocimiento de gestos de los dedos [22], [24], [34], [36], son la media, el máximo, el mínimo, la desviación estándar, la media cuadrática, el rango intercuartil y la desviación absoluta media.

Los algoritmos inteligentes de clasificación que se han evaluado en este estudio se diferencian en dos grandes grupos, algoritmos inteligentes de clasificación no lineales y combinados [4], [25].

Algoritmos inteligentes de clasificación no lineales y parámetros utilizados:

- Árbol de decisiones (Cart) con criterio de división 'gini'; estrategia de división 'best split'; hojas puras como profundidad máxima; 2 muestras mínimas para la división de un nodo interno y 1 muestra mínima en el nodo de la hoja.

- $\quad K$ vecinos más cercanos (KNN) con 'ball tree' como algoritmo de computación y 'minkowski' como métrica de distancia. En la clasificación de los movimientos de la muñeca, dedo índice y dedos medio-anular, el conjunto de datos se agrupará en $\mathrm{k}=3$ clases, uno por cada uno de las posiciones analizadas. Sin embargo, en el caso de la clasificación de los movimientos del dedo pulgar será en $\mathrm{k}=6$ clases.

- $\quad$ SVM (Support Vector Machine) con 'rbf' como kernel; penalización $\mathrm{C}$ del valor 1 y gamma del valor 0.1

- Perceptrón multicapa (Mlp) con una capa oculta de 100 neuronas; 'relu' como función de activación de la capa oculta; optimizador estocástico basado en la gradiente como optimizador de pesos; penalización L2 del valor 0.0001 y tasa de aprendizaje 'constante' en la actualización de pesos.

Algoritmos inteligentes de clasificación combinados y parámetros utilizados: 
- Random forest (Rf) [2], [14] con criterio de división 'gini'; 100 árboles; hojas puras como profundidad máxima de cada árbol; 2 muestras mínimas para la división de un nodo interno y 1 muestra mínima en el nodo de la hoja.

- Extra Trees (Et) [6] con criterio de división 'gini'; 100 árboles; hojas puras como profundidad máxima de cada árbol; 2 muestras mínimas para la división de un nodo interno y 1 muestra mínima en el nodo de la hoja.

- Agregación de bootstrap (Bagging (Bag)) [3], [7], [29] con 'árbol de decisiones' como estimador base; 10 estimadores base, y, 1 muestra máxima y 1 característica máxima extraídas del conjunto de datos para el entrenamiento de cada estimador base.

- Potenciación de gradiente (Gradient boosting $(G b m)$ ) [9], [10], [20] con una tasa de aprendizaje de cada árbol de $0.1 ; 100$ estados de potenciación; profundidad máxima de cada estimador de $3 ; 2$ muestras mínimas para la división de un nodo interno y 1 muestra mínima en el nodo de la hoja.

Los parámetros de cada algoritmo se han obtenido de manera empírica. A la hora de comparar los algoritmos en cada uno de los clasificadores mencionados anteriormente (muñeca, dedo pulgar, dedo índice y dedos medio-anular), se han tenido en cuenta tanto la precisión de la clasificación, como el tiempo de entrenamiento y evaluación que necesitan los algoritmos de clasificación. De todos los datos recogidos para el reconocimiento de los gestos de la mano, el $70 \%$ se ha utilizado para el entrenamiento y el $30 \%$ para el testeo de movimientos primitivos. Los datos obtenidos del movimiento de agarre se han utilizado para un segundo testeo, el de movimientos más complejos.

\section{RESULTADOS}

Se ha llevado a cabo una comparación para cada clasificador: muñeca, dedo pulgar, dedo índice y dedos medio-anular, en términos de precisión $\mathrm{y}$ tiempo de computación (incluyendo el entrenamiento y la evaluación).

En la fase de testeo de los movimientos primitivos, prácticamente todos los algoritmos obtienen una precisión mayor al $90 \%$ en la clasificación de cada uno de los cuatro movimientos a clasificar. Además, cabe mencionar que, a excepción de la clasificación de movimientos del dedo pulgar, se consiguen clasificaciones con precisiones superiores al $94 \%$ con todos los algoritmos. Por otro lado, al analizar el tiempo de computación entre los algoritmos que han mostrado mayor precisión, se observa que, en la clasificación de los movimientos de los dedos, el algoritmo Et se sobrepone a los demás. En el caso de la clasificación del movimiento de la muñeca se puede decir que todos los algoritmos obtienen un buen resultado, aunque los algoritmos Mlp y Gbm necesitan tiempos de computación muy altos comparando con los demás (Tabla 1).

Tabla 1: Comportamiento de los algoritmos de clasificación para movimientos de muñeca, dedo pulgar, dedo índice y dedos medio-anular en la fase de testeo de movimientos primitivos. Ordenados de mayor a menor precisión.

\begin{tabular}{|c|c|c|c|}
\hline & $\begin{array}{l}\text { Algoritmo de } \\
\text { clasificación }\end{array}$ & Precisión & $\begin{array}{c}\text { Tiempo de } \\
\text { computación(s) }\end{array}$ \\
\hline \multirow[t]{8}{*}{ Muñeca } & Mlp & 98,795 & 13,618 \\
\hline & Gbm & 98,758 & 23,904 \\
\hline & Bag & 98,722 & 1,142 \\
\hline & $\mathrm{Rf}$ & 98,649 & 1,801 \\
\hline & Et & 98,649 & 0,487 \\
\hline & KNN & 98,283 & 1,711 \\
\hline & Cart & 98,283 & 0,195 \\
\hline & SVM & 98,283 & 0,665 \\
\hline \multirow[t]{8}{*}{ Dedo índice } & $\overline{E t}$ & 98,685 & 0,81 \\
\hline & $\mathrm{Rf}$ & 98,503 & 4,082 \\
\hline & $\mathrm{Gbm}$ & 98,393 & 13,525 \\
\hline & Mlp & 98,356 & 28,805 \\
\hline & KNN & 98,064 & 17,724 \\
\hline & SVM & 97,882 & 4,921 \\
\hline & Bag & 97,443 & 6,008 \\
\hline & Cart & 96,676 & 2,657 \\
\hline \multirow{8}{*}{$\begin{array}{c}\text { Dedos medio- } \\
\text { anular }\end{array}$} & $\overline{E t}$ & 97,772 & 1,127 \\
\hline & $\mathrm{Rf}$ & 97,516 & 4,859 \\
\hline & Mlp & 96,969 & 18,444 \\
\hline & Bag & 96,713 & 4,374 \\
\hline & $\mathrm{KNN}$ & 96,676 & 3,216 \\
\hline & $\mathrm{Gbm}$ & 96,676 & 10,957 \\
\hline & SVM & 95,8 & 1,691 \\
\hline & Cart & 94,631 & 0,585 \\
\hline \multirow[t]{8}{*}{ Dedo pulgar } & $\mathrm{Et}$ & 94,777 & 0,98 \\
\hline & $\mathrm{Rf}$ & 94,229 & 3,759 \\
\hline & Gbm & 92,549 & 21,607 \\
\hline & Bag & 92,513 & 3,301 \\
\hline & Mlp & 92,367 & 22,331 \\
\hline & SVM & 91,015 & 4,122 \\
\hline & $\mathrm{KNN}$ & 90,431 & 3,117 \\
\hline & Cart & 89,481 & 0,665 \\
\hline
\end{tabular}

Habiendo obtenido unos resultados satisfactorios en la fase de testeo de movimientos primitivos, se ha analizado el comportamiento de los algoritmos de clasificación en la fase de testeo con el movimiento de agarre. 
Se puede observar (Tabla 2) que en la clasificación del comportamiento de la muñeca los algoritmos Et, SVM, KNN, Mlp y Rf obtienen precisiones superiores al 89\%, siendo el Et seguido del SVM el que menor tiempo de computación requiere (Tabla 2).

Tabla 2: Comportamiento de los algoritmos de clasificación de movimientos de muñeca, dedo pulgar, dedo índice y dedos medio-anular en la fase de testeo de movimientos de agarre. Ordenados de mayor a menor precisión.

\begin{tabular}{|c|c|c|c|}
\hline & $\begin{array}{l}\text { Algoritmo de } \\
\text { clasificación }\end{array}$ & Precisión & $\begin{array}{c}\text { Tiempo de } \\
\text { computación(s) }\end{array}$ \\
\hline \multirow[t]{8}{*}{ Muñeca } & $\mathrm{Et}$ & 94,002 & 0,36 \\
\hline & SVM & 93,908 & 0,583 \\
\hline & KNN & 90,628 & 0,55 \\
\hline & Mlp & 89,972 & 10,376 \\
\hline & $\mathrm{Rf}$ & 89,035 & 1,381 \\
\hline & $\mathrm{Gbm}$ & 76,007 & 6,024 \\
\hline & Bag & 66,448 & 0,81 \\
\hline & Cart & 47,891 & 0,161 \\
\hline \multirow[t]{8}{*}{ Dedo índice } & $\mathrm{Rf}$ & 89,128 & 3,611 \\
\hline & Et & 88,754 & 0,75 \\
\hline & $\mathrm{Gbm}$ & 88,285 & 12,928 \\
\hline & KNN & 85,754 & 1,535 \\
\hline & SVM & 85,754 & 1,2 \\
\hline & Mlp & 85,192 & 8,994 \\
\hline & Bag & 78,163 & 3,931 \\
\hline & Cart & 74,414 & 0,52 \\
\hline \multirow{8}{*}{$\begin{array}{l}\text { Dedos medio- } \\
\text { anular }\end{array}$} & Et & 83,13 & 0,58 \\
\hline & SVM & 82,662 & 1,101 \\
\hline & Mlp & 82,287 & 14,447 \\
\hline & KNN & 80,037 & 0,89 \\
\hline & $\mathrm{Rf}$ & 73,664 & 2,781 \\
\hline & $\mathrm{Gbm}$ & 73,571 & 8,254 \\
\hline & Cart & 57,357 & 0,43 \\
\hline & Bag & 55,67 & 2,291 \\
\hline \multirow[t]{8}{*}{ Dedo pulgar } & $\mathrm{Et}$ & 79,944 & 0,75 \\
\hline & $\mathrm{Rf}$ & 77,882 & 3,081 \\
\hline & KNN & 76,007 & 1,001 \\
\hline & SVM & 74,227 & 2,352 \\
\hline & Mlp & 67,385 & 20,561 \\
\hline & Gbm & 44,611 & 18,442 \\
\hline & Bag & 40,956 & 2,674 \\
\hline & Cart & 31,396 & 0,47 \\
\hline
\end{tabular}

En cuanto al dedo índice, a pesar de que el Rf, Et y Gbm muestran una precisión muy parecida, siendo el del Rf la más alta, el Et es el que menor tiempo de computación necesita. En cambio, el Gbm es el que más tiempo computacional requiere de todos los algoritmos (Tabla 2).

En los dedos medio-anular ocurre algo similar; siendo Et, SVM y Mlp los que mejor precisión muestran. El tiempo de computación del Mlp es el más alto en comparación con los demás algoritmos. Por tanto, los algoritmos más idóneos son el Et y SVM, siendo el Et ligeramente superior teniendo en cuenta tanto la precisión como el tiempo de computación (Tabla 2).

Analizando el dedo pulgar, se observa que en general es el clasificador que ha obtenido los peores resultados de precisión. Sin embargo, los algoritmos Et y Rf se sitúan cerca del $80 \%$ de precisión de clasificación. Observando el tiempo de computación, una vez más el Et ha resultado sersuperior (Tabla 2).

\section{CONCLUSIÓN Y TRABAJOS FUTUROS}

En esta comparativa preliminar se ha podido observar que la mayoría de los algoritmos han obtenido resultados satisfactorios en los cuatro clasificadores. Aun así, los movimientos realizados con el dedo pulgar han sido los más difíciles de clasificar. Una de las posibles razones de este resultado podría ser el etiquetado, ya que los distintos grados de libertad y posiciones del pulgar no han sido fácilmente perceptibles o diferenciables en algunos casos en los videos grabados. Además, el clasificador del pulgar requiere identificar 6 posiciones distintas, a diferencia del resto de clasificadores, que identifican 3 posiciones. Por otro lado, teniendo en cuenta la precisión de clasificación y el tiempo computacional de los algoritmos de clasificación, el Et, Rf y SVM han demostrado en general los mejores resultados.

Este estudio preliminar ha confirmado la capacidad de varios algoritmos de aprendizaje automático de clasificar distintas posturas de la mano y los dedos. El objetivo final es integrarlos en sistemas de control de FES en rehabilitación neurológica, por lo tanto, algunos de los trabajos a realizar en el futuro podrían ser los siguientes:

- Realizar un estudio exhaustivo de parametrización de los clasificadores que han resultado más satisfactorios en este estudio preliminar.

- Recogida de datos de una mayor muestra de voluntarios sanos y análisis de precisión intersujeto e intra-sujeto de los clasificadores.

- Recogida de datos en clínica y validación/adaptación de los clasificadores para pacientes neurológicos. 


\section{Agradecimientos}

La realización de este estudio ha sido posible gracias a la colaboración realizada entre la Universidad del País Vasco y Tecnalia por medio del marco EUSKAMPUS, concretamente en el proyecto ESKUAZ.

\section{English summary}

\section{COMPARISON OF MACHINE LEARNING ALGORITHMS FOR CLASSIFICATION OF HAND AND FINGER POSTURES}

\begin{abstract}
Upper limb impairments are one of the most common consequences of stroke. Functional electrical stimulation (FES) has proved to be an effective therapy to restore hand function and mobility. In order to develop a control system for FES applications, it is first necessary to obtain a classifier able to identify hand and fingers movements. This study contributes to the initial phase of this process, where the behaviour of different classification algorithms is analysed for classification of hand gestures during grasping movements of a healthy volunteer. Among the classification algorithms used for the recognition of hand and finger movements, the ones with the best results regarding the accuracy and computing time have been Extra Trees, Random Forest and SVM.
\end{abstract}

Keywords: Classification, machine learning, hand, fingers, inertial sensors, wearable sensors

\section{Referencias}

[1] D. Biswas et al., «On the data analysis for classification of elementary upper limb movements», Biomed. Eng. Lett., vol. 4, n. ${ }^{\circ}$ 4, pp. 403-413, 2015.

[2] L. Breiman, «Random Forests», Mach. Learn., vol. 45, n. ${ }^{\circ}$ 1, pp. 5-32, 2001.

[3] L. Breiman, «Bagging Predictors», Mach. Learn., vol. 24, n. ${ }^{\circ}$ 2, pp. 123-140, 1996.

[4] E. Bulbul, A. Cetin, y I. A. Dogru, «Human Activity Recognition Using Smartphones», en 2018 2nd International Symposium on Multidisciplinary Studies and Innovative Technologies (ISMSIT), 2018, pp. 1-6.

[5] X. Chen, X. Zhang, Z.-Y. Zhao, J.-H. Yang, V. Lantz, y K.-Q. Wang, «Hand Gesture Recognition Research Based on Surface
EMG Sensors and 2D-accelerometers», en 2007 11th IEEE International Symposium on Wearable Computers, 2007, pp. 1-4.

[6] A. K. CHOWDHURY, D. TJONDRONEGORO, V. CHANDRAN, y S. G. TROST, «Ensemble Methods for Classification of Physical Activities from Wrist Accelerometry», Med. Sci. Sport. Exerc., vol. 49, n. ${ }^{\circ}$ 9, pp. 1965-1973, sep. 2017.

[7] T. G. Dietterich, «An Experimental Comparison of Three Methods for Constructing Ensembles of Decision Trees: Bagging, Boosting, and Randomization», Mach. Learn., vol. 40, n. ${ }^{\circ}$ 2, pp. 139-157, 2000.

[8] M. Espinilla et al., «Human Activity Recognition from the Acceleration Data of a Wearable Device. Which Features Are More Relevant by Activities?», Proceedings, vol. 2, n. ${ }^{\circ}$ 19, p. 1242, oct. 2018.

[9] J. H. Friedman, «Stochastic gradient boosting», Comput. Stat. Data Anal., vol. 38, n. ${ }^{\circ} 4$, pp. 367-378, feb. 2002.

[10] J. H. Friedman, «Greedy function approximation: A gradient boosting machine.», Ann. Stat., vol. 29, n. ${ }^{\circ}$ 5, pp. 1189-1232, oct. 2001.

[11] M. Gjoreski et al., «How Accurately Can Your Wrist Device Recognize Daily Activities and Detect Falls?», Sensors, vol. 16, n. ${ }^{\circ}$ 6, p. 800, jun. 2016.

[12] G. E. Gresham et al., «Post-stroke rehabilitation: assessment, referral and patient management. Post-Stroke Rehabilitation Guideline Panel. Agency for Health Care Policy and Research.», Am. Fam. Physician, vol. 52, n. ${ }^{\circ}$ 2, pp. 461-70, ago. 1995.

[13] J. Guerra et al., «Capture, learning, and classification of upper extremity movement primitives in healthy controls and stroke patients.», IEEE Int. Conf. Rehabil. Robot., vol. 2017, pp. 547-554, 2017.

[14] T. Hastie, R. Tibshirani, y J. H. (Jerome H. . Friedman, The elements of statistical learning: data mining, inference, and prediction. Springer, 2001.

[15] H. Hefter, W. H. Jost, A. Reissig, B. Zakine, A. M. Bakheit, y J. Wissel, «Classification of posture in poststroke upper limb spasticity», Int. J. Rehabil.Res., p. 1, may 2012.

[16] Intercollegiate Stroke Working Party, National Clinical Guidelines for Stroke., vol. 4th editio. 2012.

[17] A. I. Kapandji, Fisiologia articular / miembros superiores / upper limb. Editorial Medica Panameri, 2007.

[18] K. Kisiel-Sajewicz et al., «Weakening of 
Synergist Muscle Coupling During Reaching Movement in Stroke Patients», Neurorehabil. Neural Repair, vol. 25, n. ${ }^{\circ} 4$, pp. 359-368, may 2011.

[19] M. F. Levin, R. W. Selles, M. H. Verheul, y O. G. Meijer, «Deficits in the coordination of agonist and antagonist muscles in stroke patients: implications for normal motor control.», Brain Res., vol. 853, n. ${ }^{\circ}$ 2, pp. 35269, ene. 2000.

[20] L. Mason, J. Baxter, P. Bartlett, y M. Frean, «Boosting algorithms as gradient descent», Proceedings of the 12th International Conference on Neural Information Processing Systems. MIT Press, pp. 512-518, 1999.

[21] F. Molina Rueda, F. M. Rivas Montero, M. Pérez de Heredia Torres, I. M. Alguacil Diego, A. Molero Sánchez, y J. C. Miangolarra Page, «Análisis del movimiento de la extremidad superior hemiparética en pacientes con accidente cerebrovascular: estudio piloto», Neurología, vol. 27, n. ${ }^{\circ}$, pp. 343-347, jul. 2012.

[22] A. Moschetti, L. Fiorini, D. Esposito, P. Dario, y F. Cavallo, «Toward an Unsupervised Approach for Daily Gesture Recognition in Assisted Living Applications», IEEE Sens. J., vol. 17, n. ${ }^{\circ}$ 24, pp. 8395-8403, dic. 2017.

[23] A. Moschetti, L. Fiorini, D. Esposito, P. Dario, y F. Cavallo, «Recognition of Daily Gestures with Wearable Inertial Rings and Bracelets.», Sensors (Basel)., vol. 16, n. ${ }^{\circ} 8$, ago. 2016.

[24] A. Moschetti, L. Fiorini, D. Esposito, P. Dario, y F. Cavallo, «Recognition of Daily Gestures with Wearable Inertial Rings and Bracelets.», Sensors (Basel)., vol. 16, n. ${ }^{\circ} 8$, ago. 2016.

[25] S. Mysore, «Human Activity Recognition from Sensor Data». 2018.

[26] M. B. Popovic, D. B. Popovic, T. Sinkjær, A. Stefanovic, y L. Schwirtlich, «Restitution of Reaching and Grasping Promoted by Functional Electrical Therapy», Artif. Organs, vol. 26, n. ${ }^{\circ}$ 3, pp. 271-275, mar. 2002.

[27] N. Ravi, N. Dandekar, P. Mysore, y M. L. Littman, «Activity recognition from accelerometer data», Proc. 17th Conf. Innov. Appl. Artif. Intell. - Vol. 3, pp. 1541-1546, 2005.

[28] C. C. Silva et al., «Co-activation of upper limb muscles during reaching in post-stroke subjects: An analysis of the contralesional and ipsilesional limbs», J. Electromyogr. Kinesiol., vol. 24, n. ${ }^{\circ}$ 5, pp. 731-738, oct. 2014.
[29] M. Skurichina y R. P. W. Duin, «Bagging, Boosting and the Random Subspace Method for Linear Classifiers», Pattern Anal. Appl., vol. 5, n. ${ }^{\circ}$ 2, pp. 121-135, jun. 2002.

[30] D. K. Sommerfeld, E. U.-B. Eek, A.-K. Svensson, L. W. Holmqvist, y M. H. von Arbin, «Spasticity After Stroke - Its Occurrence and Association With Motor Impairments and Activity Limitations», Stroke, vol. 35, n. ${ }^{\circ}$ 1, pp. 134-139, ene. 2004.

[31] O. Steven Eyobu y D. S. Han, «Feature Representation and Data Augmentation for Human Activity Classification Based on Wearable IMU Sensor Data Using a Deep LSTM Neural Network.», Sensors (Basel)., vol. 18, n. ${ }^{\circ}$, ago. 2018.

[32] R. Teasell et al., «EVIDENCE-BASED REVIEW OF STROKE REHABILITATION», vol. 18th Editi, n. ${ }^{\circ}$ December, pp. 1-66, 2016.

[33] M. Verleysen, A. Ghio, L. Oneto, X. Parra Perez, y J. L. Reyes Ortiz, A Public Domain Dataset for Human ActivityRecognition Using Smartphones. 2013.

[34] H. Wen, J. Ramos Rojas, y A. K. Dey, «Serendipity: Finger Gesture Recognition usingan Off-the-Shelf Smartwatch», en Proceedings of the 2016 CHI Conference on Human Factors in Computing Systems - CHI '16, 2016, pp. 3847-3851.

[35] J. Wu, L. Sun, y R. Jafari, «A Wearable System for Recognizing American Sign Language in Real-Time Using IMU and Surface EMG Sensors», IEEE J. Biomed. Heal. Informatics, vol. 20, n. ${ }^{\circ}$ 5, pp. 12811290, 2016.

[36] Y. Zhou, L. Jing, y Z. Cheng, "Automatic Recognition of Finger Gestures Using a TriAxis Accelerometer», Int. J. Comput. Theory Eng., pp. 967-970, 2013.

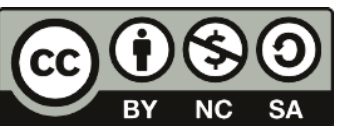

(C) 2019 by the authors. Submitted for possible open access publication under the terms and conditions of the Creative Commons Attribution CC BY-NC-SA 4.0 license (https://creativecommons.org/licenses/bync-sa/4.0/deed.es). 